\title{
Polarization modulation instability in a Manakov fiber system
}

\author{
B. Frisquet, ${ }^{1}$ B. Kibler, ${ }^{1,}{ }^{*}$ J. Fatome, ${ }^{1}$ P. Morin,,${ }^{1}$ F. Baronio, ${ }^{2}$ M. Conforti, ${ }^{3}$ G. Millot,,${ }^{1}$ and S. Wabnitz ${ }^{2}$ \\ ${ }^{1}$ Laboratoire Interdisciplinaire Carnot de Bourgogne (ICB), UMR 6303 CNRS-Université de Bourgogne Franche-Comté, \\ F-21078 Dijon, France \\ ${ }^{2}$ INO CNR and Department of Information Engineering, Università di Brescia, Brescia, Italy \\ ${ }^{3}$ Univ. Lille, CNRS, UMR 8523 - PhLAM - Physique des Lasers Atomes et Molécules, F-59000 Lille, France
}

(Received 14 September 2015; published 23 November 2015)

\begin{abstract}
The Manakov model is the simplest multicomponent model of nonlinear wave theory: It describes elementary stable soliton propagation and multisoliton solutions, and it applies to nonlinear optics, hydrodynamics, and Bose-Einstein condensates. It is also of fundamental interest as an asymptotic model in the context of the widely used wavelength-division-multiplexed optical fiber transmission systems. However, although its physical relevance was confirmed by the experimental observation of Manakov (vector) solitons in a planar waveguide in 1996, there have in fact been no quantitative experiments confirming its validity for nonlinear dynamics other than soliton formation. Here, we report experiments in optical fiber that provide evidence of passband and baseband polarization modulation instabilities in a defocusing Manakov system. In the spontaneous regime, we also reveal a unique saturation effect as the pump power increases. We anticipate that such observations may impact the application of this minimal model to describe and understand more complicated phenomena in nature, such as the formation of extreme waves in multicomponent systems.
\end{abstract}

DOI: 10.1103/PhysRevA.92.053854

PACS number(s): 42.65.-k, 42.81.-i

\section{INTRODUCTION}

In complex wave systems, we have to deal with more than only one variable (i.e., more than a single wave amplitude), which often requires multicomponent generalizations of a single nonlinear partial differential equation. For instance, consider a system of coupled nonlinear Schrödinger equations (NLSEs) to describe the nonlinear interaction between wave packets in dispersive conservative media. Such coupled systems are of physical relevance in various domains such as nonlinear optics, hydrodynamics, plasma physics, multicomponent Bose-Einstein condensates, and financial systems [1-5]. The first multicomponent NLSE type of model with applications to physics is the well-known Manakov model [6]. For two components, the corresponding set of two coupled NLSEs is completely integrable through a special choice of the nonlinear parameters, thus providing suitable conditions for soliton propagation. In this context, the mathematical properties of such a model have been investigated in detail, since solitons and their interactions in multicomponent systems represent a fascinating topic in many fields of physics $[7,8]$. In particular, when considering the two coupled orthogonally polarized components of the optical field, the Manakov system admits trivial two-component stable solutions formed by two incoherent bright (self-focusing case) or dark (self-defocusing case) solitons. More generally, the Manakov system has attracted enormous attention as it is considered to be a promising model for the description of interactions between wavelengthdivision-multiplexed (WDM) channels of long optical fiber transmission systems [9], polarization-division-multiplexed systems (PDM) [10,11], or even space-division-multiplexed (SDM) systems using multimode or multicore fibers [12].

\footnotetext{
*Corresponding author: FAX: +33380395971, bertrand.kibler@ubourgogne.fr
}

A sufficient condition for the integrability of the two coupled NLSEs is the following: The dispersion coefficient should be the same for both modes; moreover, the ratio between the self-phase modulation (SPM) and the cross-phase modulation (XPM) has to be equal to unity, and the SPM coefficients need to be equal for the two components $[6,13]$. Moreover, one has to check that the coupled wave system does not exhibit any coherent nonlinear energy exchange between components, also known as four-wave mixing (FWM) [14]. Theoretical studies have predicted that such requirements are well statisfied when considering the orthogonally polarized components of a vector field propagating in standard optical fibers exhibiting a rapidly varying, low birefringence, as those which are typically used nowadays for fiber-based telecommunication systems $[15,16]$. Indeed, random changes in fiber birefringence occur on a very short length scale when compared with the nonlinear length in optical transmission links, so that an average nonlinear effect can be approximated, thus reducing the cross-phase modulation coefficient to unity, in contrast to the case of homogeneous birefringent fibers. However, the first experimental observation of Manakov (vector) solitons in a strict mathematical sense has been reported in the spatial domain with crystals by Kang et al. [17]. In that work, the authors arranged Manakov conditions in real cubic crystals by using TE and TM polarizations in an AlGaAs planar waveguide operating at a wavelength below half its band gap. Despite extensive mathematical studies [18-21], experiments have been limited to only a small number of systems such as optical waveguides and Bose-Einstein condensates [22-24]. There is a need for more studies to quantitatively characterize nonlinear wave propagation described by the Manakov model, besides the soliton evidence and its qualitative application to the design of optical networks [16,25], and a vector extension of the well-known modulation instability (MI) in the anomalous dispersion regime of telecom fibers, leading to high-repetition-rate pulse trains [26]. As a result, the peculiar nonlinear dynamics and the associated instabilities $[27,28]$ that 
can be predicted by using the Manakov model have remained so far largely quantitatively untested.

In this contribution, we point out and experimentally demonstrate that fundamental nonlinear instabilities can be quantitatively well predicted by the Manakov model when using relatively long spans of randomly birefringent, lowpolarization mode dispersion (PMD) telecom fibers. We analyze the particular case of polarization modulation instability (PMI) in the normal dispersion regime (i.e., the defocusing regime in the equivalent spatial case), which can be of a passband or baseband nature [29,30]. The MI of two WDM pumps with an orthogonal state of polarization is induced by spontaneous (or quantum) noise. Wave propagation is governed by two incoherently coupled nonlinear Schrödinger equations of the Manakov type. In the spontaneous MI regime, we reveal a saturation effect of the peak gain, and verify the predicted existence of an optimum frequency of the modulation instability as the pump power increases.

This work is organized as follows. In Sec. II, we first provide a theoretical analysis describing PMI in a defocusing Manakov system. In Sec. III, we describe our experimental setup for observing PMI. Next, we present the experimental results of PMI in the spontaneous regime, and compare them with numerical simulations based on the coupled system of nonlinear Schrödinger equations of Manakov type. In Sec. IV, we conclude this work.

\section{THEORETICAL ANALYSIS OF POLARIZATION MODULATION INSTABILITY}

The coupling between two laser beams in nonlinear dielectric media may lead to a large and diverse set of fascinating physical effects. Typical examples of such effects are FWM and MI processes. In optical fibers, the conservative interaction between nonlinear and dispersive effects leads to phenomena such as MI, whereby a continuous or quasicontinuous wave undergoes a modulation of its amplitude or phase in the presence of noise or any other weak perturbation [14,31-34]. The perturbation can originate from quantum noise (spontaneous MI) or from a weak frequency shifted signal wave (induced MI). MI has been observed for the first time for a single pump wave propagating in a standard nonbirefringent fiber (scalar MI) [33]. In this framework, it was shown that scalar MI only occurs in the focusing regime, or in the anomalous group-velocity dispersion (GVD) regime. However, the extension of MI to the normal dispersion regime was first pointed out by Berkhoer and Zakharov, by considering the nonlinear coupling between two different (e.g., polarization) modes via XPM [34]. Subsequently, several experiments with two polarization modes were performed in order to observe cross-polarization modulation (XPolM) in birefringent fibers [35-37]. It has long ago been predicted that XPM-induced MI should also be observable in the normal GVD regime thanks to the nonlinear interaction among two different WDM pumps with parallel states of polarization [38], but the process can be hampered by the simultaneous presence of FWM [39-41]. The situation is completely different when two WDM pumps with orthogonal states of polarization are involved [37]: Whenever the group-velocity mismatch between the two orthogonal modes is large, the XPolM-
MI gain is relatively narrowband, and the FWM among orthogonally polarized pumps is effectively suppressed. In all of the previous experiments on MI, fiber birefringence was such that the ratio between SPM and XPM was different from unity, which does not correspond to the coupled system of Manakov type. However, this condition should be well statisfied when studying orthogonally polarized components of a vector field propagating in standard optical fibers exhibiting a rapidly varying low birefringence, as it occurs in the fibers which are typically used nowadays for long-distance optical communication links. From the point of view of telecom applications, XPolM-MI can be an essential source of nonlinear impairments for WDM systems with polarization interleaving or polarization multiplexing [42-45]. Also on a more fundamental level, MI is a generic nonlinear process that occurs in many areas, and it is at the origin of very important fundamental phenomena, such as optical soliton generation [46,47], supercontinuum generation [48], and rogue wave formation [29,30,49-51].

The propagation of two orthogonally polarized optical pump waves at a relative frequency offset, say, $\Delta=\Delta \omega / 2 \pi$, in the normal dispersion regime of a randomly birefringent telecom fiber with relatively low-polarization mode dispersion is described in terms of the two incoherently coupled nonlinear Schrödinger equations (CNLSEs):

$$
\begin{aligned}
& i \frac{\partial u}{\partial z}+i \frac{\delta}{2} \frac{\partial u}{\partial t}-\frac{\beta_{2}}{2} \frac{\partial^{2} u}{\partial t^{2}}+\gamma\left(|u|^{2}+|v|^{2}\right) u=0, \\
& i \frac{\partial v}{\partial z}-i \frac{\delta}{2} \frac{\partial v}{\partial t}-\frac{\beta_{2}}{2} \frac{\partial^{2} v}{\partial t^{2}}+\gamma\left(|v|^{2}+|u|^{2}\right) v=0 .
\end{aligned}
$$

Equation (1) is equivalent to the Manakov system $[6,15,16]$, once the group-velocity mismatch term is removed by the transformation $u(z, t)=\tilde{u}(z, t) \exp \left[i \frac{\delta}{\left(2 \beta_{2}\right)} t-i \frac{\delta^{2}}{\left(8 \beta_{2}\right)} z\right], v(z, t)=$ $\tilde{v}(z, t) \exp \left[-i \frac{\delta}{\left(2 \beta_{2}\right)} t-i \frac{\delta^{2}}{\left(8 \beta_{2}\right)} z\right]$. In Eq. (1) $z$ and $t$ denote the propagation distance and retarded time (in a reference frame traveling at the group velocity evaluated at the central carrier frequency $\omega_{0}$ ) coordinates; $u$ and $v$ are the complex slowly varying amplitudes of the two pump waves at frequencies $\omega_{\mathrm{u}, \mathrm{v}}=\omega_{\mathrm{o}} \pm \pi \Delta$, respectively; and $\delta$ is associated with their group-velocity mismatch (GVM), owing to normal groupvelocity dispersion. In fact, the $u(v)$ pump is a slow (fast) wave with respect to the waves at the carrier frequency $\omega_{0}$. $\beta_{2}$ and $\gamma$ are the group-velocity dispersion and the effective Kerr nonlinear coefficient at frequency $\omega_{0}$, respectively. Note that in Eq. (1) the variations of $\beta_{2}$ and $\gamma$ as a function of frequency are neglected, which is reasonable for the frequency detunings that are considered in our experiments $(\Delta \leqslant 500 \mathrm{GHz})$. The GVM parameter is then simply defined as $\delta=\beta_{2} \Delta$, whereas the nonlinear coefficient is written as $\gamma=\frac{8 n_{2} \omega_{o}}{9 c A_{e f f}}$, where $c$ is the light velocity in vacuum, $n_{2}=2.6 \times 10^{-20} \mathrm{~m}^{2} / \mathrm{W}$ is the nonlinear index coefficient, and $A_{\text {eff }}$ designates the effective core area at the carrier angular frequency $\omega_{0}$. The factor $8 / 9$ takes into account random variations of the intrinsic fiber birefringence [16]. We would like to emphasize the fact that coherent coupling terms [16] have been neglected in Eq. (1), since they only play a role in a very small parameter range (small values of $\Delta$ ), which is outside the operating conditions considered in our experimental study. On the other hand, we 
would like to mention that Raman effects are not considered in Eq. (1), since our experimental conditions were selected in a manner that Raman scattering is negligible. The validity of Eq. (1) in representing the MI of polarized waves or $\mathrm{PMI}$ in a randomly birefringent, low-PMD fiber was recently qualitatively confirmed in the anomalous GVD regime [26].

The linear stability analysis (LSA) of the steady-state solution of Eq. (1) leads to the eigenvalue equation $[M][Y]=$ $K[Y]$, with the eigenvector defined as $[Y]^{T}=\left[u_{a}, u_{s}^{*}, v_{a}, v_{s}^{*}\right]$, where $u_{s}$ and $u_{a}$ are the amplitudes of the Stokes and antiStokes sidebands for the pump wave of amplitude $u$ (more details about LSA can be found in Ref. [37]), whereas $v_{s}$ and $v_{a}$ represent the Stokes and anti-Stokes sidebands for the pump wave of amplitude $v$. Throughout this work we assume that the power $P$ injected into the fiber is equally distributed among the two pumps, i.e., $|u(z=0, t)|^{2}=|v(z=0, t)|^{2}=$ $P / 2$. The stability matrix of the system $[M]$ is then defined as

$$
[M]=\left(\begin{array}{cccc}
-a+b+p / 2-k & p / 2 & p / 2 & p / 2 \\
-p / 2 & -a-b-p / 2-k & -p / 2 & -p / 2 \\
p / 2 & p / 2 & a+b+p / 2-k & p / 2 \\
-p / 2 & -p / 2 & -p / 2 & a-b-p / 2-k
\end{array}\right)
$$

with $a=\pi \Delta \beta_{2} \Omega, b=\frac{1}{2} \beta_{2} \Omega^{2}$, and $p=\gamma P$, where $\Omega$ is the angular frequency of the perturbation related to the wave number $K$ by the dispersion relation $\operatorname{det}([M]-K[I])=0$. The MI phenomenon occurs when the wave number $K$ of the perturbation possesses a nonzero imaginary part, and manifests itself with an exponential growth of the amplitude of the perturbation, whose importance is measured by a power gain coefficient $G$ defined by $G(\Omega)=2|\operatorname{Im}(K)|$. From (2) one obtains the following dispersion relation:

$$
K^{2}=a^{2}+b^{2}+b p-\sqrt{b\left[4 a^{2}(b+p)+b p^{2}\right]},
$$

which yields the condition for MI to occur, that is,

$$
\max \left[0,\left(\Delta^{2}-\frac{p}{\pi^{2} \beta_{2}}\right)\right] \leqslant\left(\frac{\Omega}{2 \pi}\right)^{2} \leqslant \Delta^{2} .
$$

Figures 1(a)-1(d) show the gain spectrum for different powers, the peak gain vs total pump power $P$, the optimum modulation frequency (OMF) defined as the frequency at which the gain is maximum, and the intensities of the eigenvectors of the system, respectively. These figures have been obtained for the following parameter values: $\gamma=2.4 \mathrm{~W}^{-1} \mathrm{~km}^{-1}$, and $\beta_{2}=18 \mathrm{ps}^{2} \mathrm{~km}^{-1}$ [or $D=-14 \mathrm{ps}(\mathrm{nm} \mathrm{km})^{-1}$ ]. As can be seen from Fig. 1(a), whatever the input power, the high cutoff frequency is fixed and it remains equal to the pump spacing, whereas the low cutoff frequency depends on the input power level. $P_{o}=\frac{9 \pi^{2} \Delta^{2} \beta_{2}}{8 \gamma}$ denotes the power for which the low cutoff frequency vanishes (e.g., $P_{o}=0.134 \mathrm{~W}$ for $\Delta=40 \mathrm{GHz}$ ). One remarkable point is that in the defocusing case, contrary to the focusing case, distinct passband and baseband MI regimes exist: the bandwidth of PMI $0 \leqslant \Omega_{1}<\Omega<\Omega_{2}$, in which $\mathrm{G}(\Omega) \neq 0$, is passband if $\Omega_{1}>0$, while it is baseband if $\Omega_{1}=0$ [29,30]. Another noticeable point is that, contrary to the highly birefringent fiber case [37], there is no critical power beyond which the PMI disappears. To the contrary, in the present case there is a monotonic increase of the peak gain with input power, and a gain saturation as the power grows above about $10 P_{o}$ [see Fig. 1(b)]. The asymptotic solution to the dispersion relation [Eq. (3)] that is found in the limit of large values of $p$ leads to the saturation value of the peak gain $G_{\text {sat }}=2 \pi^{2} \Delta^{2} \beta_{2}$ and to the OMF $f_{\text {sat }}=\frac{\Delta}{\sqrt{2}}$. Figure 1(c) shows that the OMF decreases monotonically to attain the asymptotic minimum value $f_{\text {sat }}$ as $P$ increases $(\mathrm{OMF} / \Delta \rightarrow 1 / \sqrt{2}=0.707)$. The eigenvectors of the system, which are shown in Fig. 1(d), reveal that the PMI generates two dominant sidebands: an anti-Stokes sideband $u_{a}$ at frequency $\omega_{u}+\Omega$ polarized along the pump $\omega_{u}$, and a Stokes sideband $v_{s}$ at frequency $\omega_{v}-\Omega$ polarized along the pump $\omega_{v}$. However, as the input pump power increases, we can see the appearance of two secondary sidebands: a Stokes sideband $u_{s}$ at frequency $\omega_{u}-\Omega$ polarized along the pump $\omega_{u}$, and an anti-Stokes sideband $v_{a}$ at frequency $\omega_{v}+\Omega$ polarized along the pump $\omega_{v}$. Also note that the intensities of the four sidebands saturate at high power levels with an asymptotic value of the intensity of the secondary sidebands of about $16 \%$ that of the main sidebands.
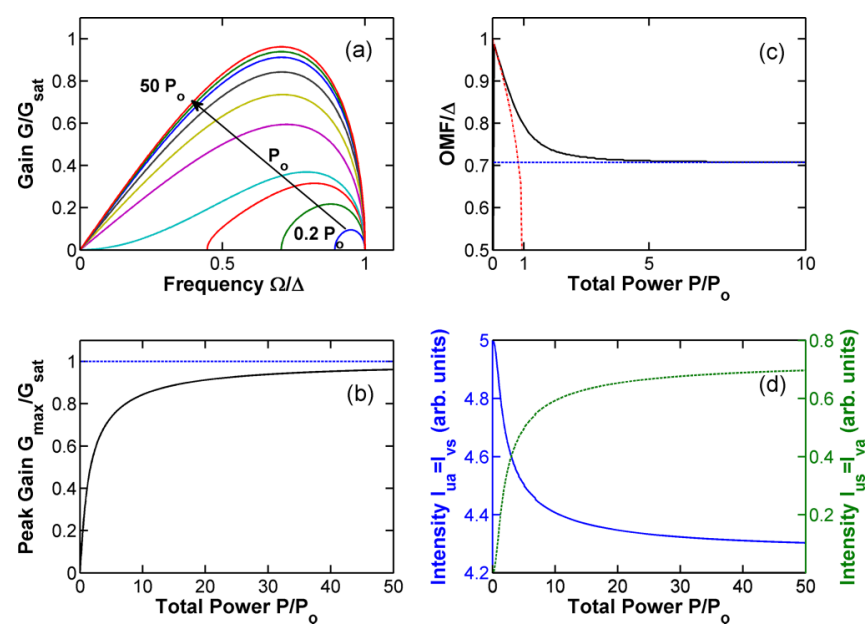

FIG. 1. (Color online) (a) PMI gain vs sideband detuning for total pump powers in the range $0.2 P_{0}-50 P_{0}$. (b) PMI peak gain vs power (the blue dashed line represents the saturation level of the peak gain). (c) OMF vs total pump power. The black line gives the results obtained from the linear stability analysis [dispersion relation (3)], whereas the red dash-dotted line is obtained via the phase-matching condition (5). The blue dashed line represents the saturation limit. (d) Intensities of the MI sidebands obtained from the eigenvectors of the system vs total pump power. The blue solid (green dashed) line corresponds to the primary (secondary) sidebands. 
Some of the previously described features can also be understood by considering the PMI phenomenon as a four-photon mixing process $\omega_{u}+\omega_{v}-\left(\omega_{u}-\Omega\right)-\left(\omega_{v}+\Omega\right)$, whereby two pump photons are transferred to the two prominent symmetric sidebands whose frequencies are given by the following phase-matching condition:

$$
\Delta k_{L}+\Delta k_{N L}=-2 \pi \Delta \beta_{2} \Omega+\beta_{2} \Omega^{2}+\gamma P=0 .
$$

The first term of the right-hand side of Eq. (5) represents the role of the GVM between the two pumps; the second term describes the role of GVD, and the last term represents the role of the Kerr nonlinearity. Since in optical fibers the nonlinear coefficient is positive (because of the selffocusing nature of the Kerr effect in glass), the phase-matching condition (5) requires a negative linear phase mismatch. Let us remark from Eq. (5) that, as the pump detuning $\Delta$ vanishes (single-frequency regime), MI only occurs for negative GVD (anomalous dispersion). In the general case of two WDM pumps $(\Delta \neq 0)$, Eq. (5) leads to complex values of the detuning $\Omega$ for pump powers $P>P_{o}$. Whenever $P<P_{o}$, Eq. (5) provides an analytical expression of the OMF:

$$
f_{\mathrm{opt}}=\frac{\Delta}{2}\left(1+\sqrt{1-\frac{P}{P_{o}}}\right) .
$$

The corresponding graph is represented by the red (dashdotted) line in Fig. 1(c). Note that the analytical expression of Eq. (6) provides a good approximation of the OMF at low pump powers (typically $P / P_{o}<0.5$ ), but it significantly deviates from the exact OMF which can be derived from the Manakov equations [Eq. (1)] at larger values of the pump power $\left(0.5<P / P_{o}<1\right)$. This discrepancy can be explained by the influence of the non-phase-matched waves (secondary pair of sidebands) [37] [see Fig. 1(d)].

\section{EXPERIMENTS ON THE SPONTANEOUS NOISE-DRIVEN PMI}

The experimental setup that we used for observing the Manakov PMI in the normal dispersion regime of a telecom fiber span is shown in Fig. 2(a). Two continuous pump waves were generated from two laser diodes emitting polarized light at different wavelengths $\lambda_{1}$ and $\lambda_{2}$, which can be tuned around the central wavelength $\left(\lambda_{0}=1554.7 \mathrm{~nm}\right)$ with a frequency detuning $\Delta$ ranging from 50 to $500 \mathrm{GHz}$. The two pump waves were superposed by means of a polarization maintaining fiber optic coupler with a 50:50 coupling ratio. In order to significantly increase the peak pump powers involved in our experiment, we temporally carved both pump waves thanks to an electro-optic modulator driven by a pseudorandom binary sequence generator. More precisely, 100-ns square pulse trains were generated with a duty cycle of $1: 10$. We would like to emphasize that in our experiments such pulses provide a quasi-cw condition for the demonstration of the MI phenomenon, since the pulse duration is about four orders of magnitude larger than the expected modulation period. Moreover, to suppress stimulated Brillouin scattering (SBS) that may occur in the optical fiber, a phase modulator was inserted into the setup in order to increase the spectral linewidth of the two pump waves. The phase modulator was driven by a (a)
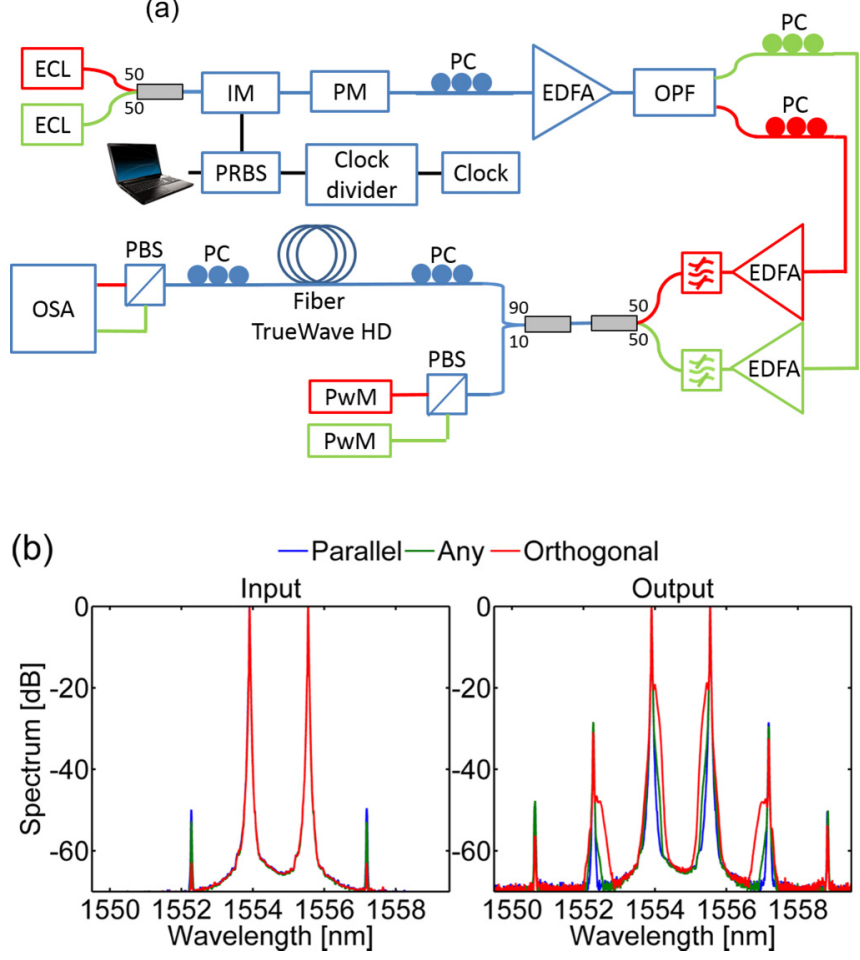

FIG. 2. (Color online) (a) Experimental setup: red and green lines depict the two wavelength-division-multiplexed and orthogonally polarized pumps. ECL: external-cavity diode laser; 50/50 and 90/10: fiber couplers; EDFA: erbium-doped fiber amplifier; IM: intensity modulator; PC: polarization controller; PM: phase modulator; PBS: polarization beam splitter; PRBS: pseudorandom binary sequence generator; PwM: power-meter; OPF: optical programmable filter; OSA: optical spectrum analyzer. (b) Impact of the input polarization states of the two-pump system with $200 \mathrm{GHz}$ frequency spacing on the observation of PMI. (Left: input spectra. Right: output spectra.)

67-MHz rf signal, thus enabling us to work at relatively high pump powers, while still being far below the SBS threshold. At this stage, the two pump waves were spectrally separated by means of a programmable optical filter (Finisar WaveShaper), while a pair of polarization controllers (PCs) allowed us to obtain two orthogonal linear states of polarization. The two pump waves were finally recombined after their independent amplification by high-power erbium-doped fiber amplifiers before injection into the optical fiber. The optical fiber used in our experiment was a reverse TrueWave fiber with a length of $5 \mathrm{~km}$, a chromatic dispersion of $-14 \mathrm{ps} / \mathrm{nm} / \mathrm{km}$, a nonlinear coefficient $\gamma=2.4 \mathrm{~W}^{-1} \mathrm{~km}^{-1}$, and the attenuation of $0.25 \mathrm{~dB} / \mathrm{km}$ at $\lambda_{0}=1554.7 \mathrm{~nm}$. This fiber has the very low nominal PMD of $0.017 \mathrm{ps} \mathrm{km}^{-1 / 2}$. At the fiber output, a polarization beam splitter (PBS) selected the output light propagating in the two orthogonal linear polarizations. The two resulting waves were simultaneously analyzed in the spectral domain by means of two optical spectrum analyzers (OSAs). Note that the orthogonal polarization states were continuously tracked before injection into the fiber thanks to power measurements after their separation with a polarization beam splitter. To highlight the fundamental impact of the initial polarization state of the two-pump system on the observation of 
(a) CNLSE U-axis

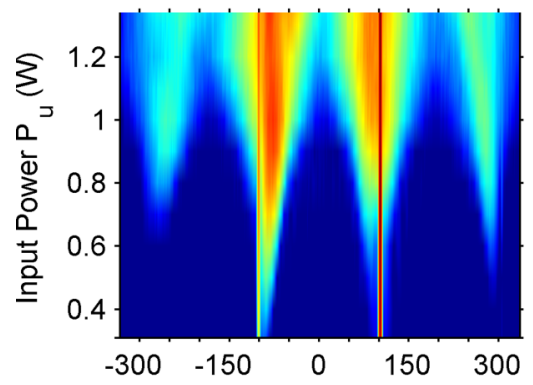

(b) CNLSE V-axis

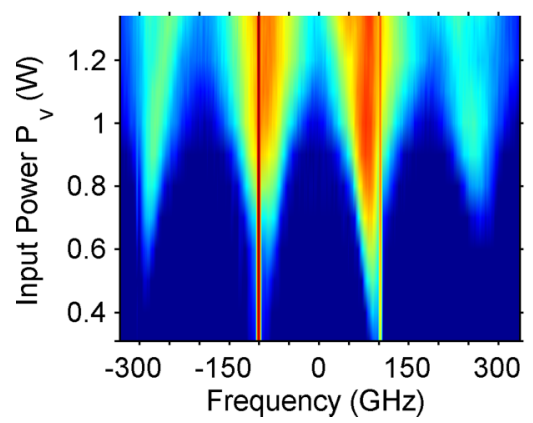

(c) Expt. U-axis

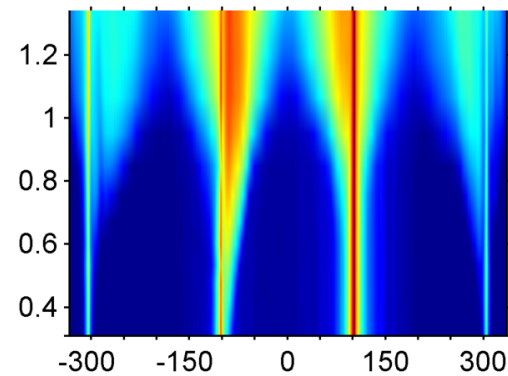

(d) Expt. V-axis

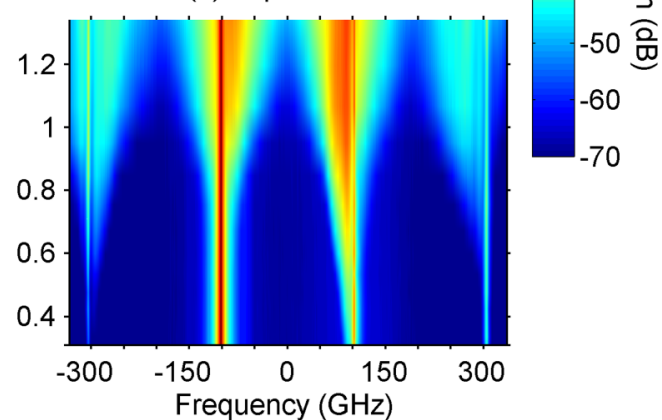

FIG. 3. (Color online) Output spectra vs input pump power on $u$ and $v$ axis. (a,b) numerical simulation of the CNLSEs (1) with loss; (c,d) experiments.
PMI, Fig. 2(b) reports spectral measurements both at the fiber input and at the fiber output for three distinct configurations of the relative polarization of the input pumps (parallel, any, and orthogonal). Our results clearly confirm that PMI was only observed in the single case of orthogonally polarized pumps, as predicted by our theory. Note that some narrow FWM sidebands cannot be completely suppressed before injection into the telecom fiber, thus leading to their subsequent amplification upon propagation.

Figure 3 compares the numerical output spectra emerging from the two orthogonal polarization states of the fiber (left column) with the experimental spectra, as a function of the input power $P_{u}$ and $P_{v}$ of the two pumps, which are linearly polarized along orthogonal $u$ and $v$ directions of the fiber, respectively. Note that the orientation of these polarization directions is completely arbitrary, since the fiber is nominally circular; hence it does not exhibit two principal axes of polarization. Here the frequency spacing between the two pumps was set to $200 \mathrm{GHz}$, corresponding to $P_{o}=3.3 \mathrm{~W}$ and $G_{\text {sat }}=62 \mathrm{~dB} / \mathrm{km}$. We consider the passband PMI regime. As it can be seen from Fig. 3, for pump powers above $0.5 \mathrm{~W}$, a broadband PMI spectrum develops as a mirror (and orthogonally polarized) image of each pump. Figure 3 reveals the excellent agreement between the numerical solutions of the CNLSEs (1) with added loss and the experimental results. Moreover, Fig. 3 shows the emergence of secondary outer sidebands, as predicted by the linear stability analysis at the highest powers as $P=2 P_{u}=2 P_{v}$ approaches $P_{o}$. Note that the growth of these secondary sidebands is reinforced by the cascaded FWM between each pump and the parallel primary PMI sideband. We can also observe from Fig. 3 that the gravity center of the primary sideband approaches the pump as the pump power increases, in good qualitative agreement with the analytical predictions of the linear stability analysis.
A more precise quantitative assessment of the agreement among theory and experiments is provided by Fig. 4, where we compare the cumulated PMI gain as a function of the input pump power $P_{\text {tot }}$, for both the primary and the secondary sidebands, as predicted by the CNLSEs (solid curves) and by the experiments (empty dots). Figure 4 also shows (with a set of stars) the analytical PMI gain obtained from the linear stability analysis of Eq. (1). The numerical and experimental gains are both reduced with respect to the analytical predictions, since a nonlinear saturation of the PMI gain occurs for powers above $1.5 \mathrm{~W}$. The observed PMI gain saturation is due to the influence of FWM between each pump and the parallel primary PMI sideband, as well as to pump depletion.

We also experimentally studied the dependence of PMI gain upon the frequency spacing among the two pumps.

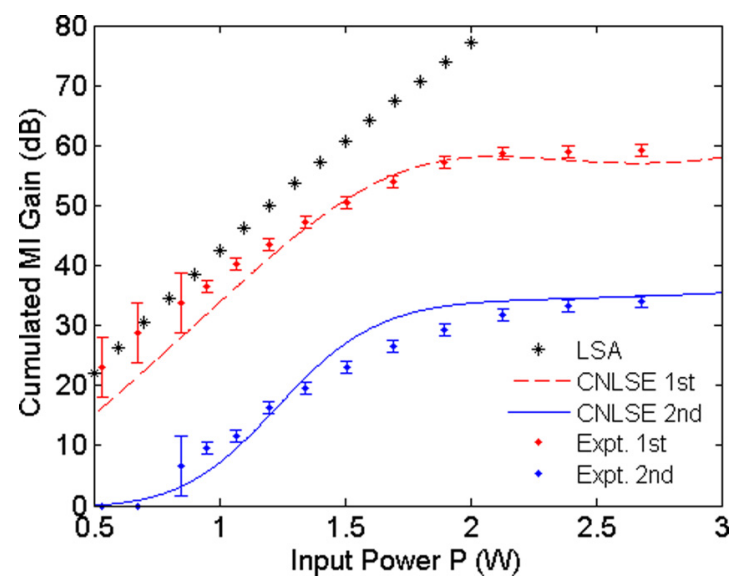

FIG. 4. (Color online) Dependence of cumulated PMI gain (primary and secondary sidebands) on input total pump power. 

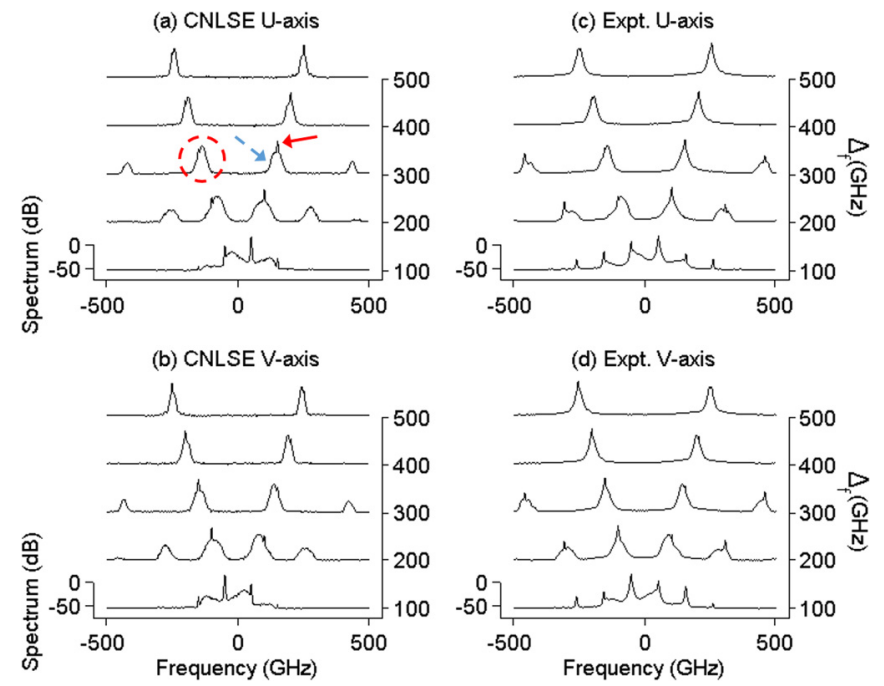

FIG. 5. (Color online) Variation of output spectra as a function of the pump frequency spacing. (a,b): numerical simulation of the CNLSEs (1) with loss; $(\mathrm{c}, \mathrm{d})$ : experiments. The emergence of the mirror image of the MI spectrum is revealed in subfigure (a) as the broad pedestal indicated by blue dashed arrow around the pump spectrum (red arrow). The primary MI sideband is highlighted by the red dashed circle.

Figure 5 compares numerical (left column) and experimental output spectra (right column) emerging from orthogonal polarizations, as the pump spacing $\Delta$ is decreased from $500 \mathrm{GHz}\left(P_{o}=20.8 \mathrm{~W}, G_{\text {sat }}=386 \mathrm{~dB} / \mathrm{km}\right)$ up to $100 \mathrm{GHz}$ $\left(P_{o}=0.837 \mathrm{~W}, G_{\mathrm{sat}}=15.4 \mathrm{~dB} / \mathrm{km}\right)$. Hereafter the pump powers are fixed to $0.8 \mathrm{~W}$ on each fiber axis (total pump power $\left.P=2 P_{u}=2 P_{v}=1.6 \mathrm{~W}\right)$. Figure 5 clearly reveals the existence of the passband PMI regime (for $200 \mathrm{GHz} \leqslant \Delta \leqslant$ $500 \mathrm{GHz})$ and of the baseband MI regime $(\Delta=100 \mathrm{GHz})$. It is important to note that the existence of a baseband MI regime is closely linked with the mechanism leading to rogue wave formation [29]. Besides the excellent quantitative agreement between theory and experiments, Fig. 5 also reveals, for pump frequency spacings around $200-300 \mathrm{GHz}$, the unexpected emergence of a mirror (or frequency-conjugate) image of the MI spectrum which appears as a broad pedestal around each (relatively narrowband) pump spectrum.

A quantitative overview of the agreement between the analytical PMI gain predictions resulting from the linear stability analysis of Eq. (1), their full numerical solutions including a weak noise seed and loss, and the experiments is illustrated in Fig. 6. As it can be seen, the analytical PMI gain predictions are in good quantitative agreement with both simulations and experiments for pump detunings below $150 \mathrm{GHz}$ only. In fact, the PMI gain saturates slightly above $40 \mathrm{~dB}$ because of the transfer of power into the secondary sidebands, owing to cascade FWM.

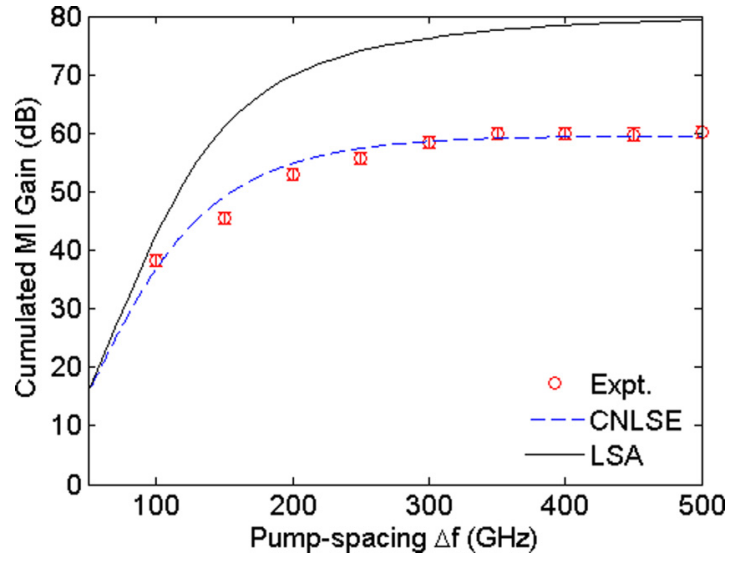

FIG. 6. (Color online) Comparison between analytical predictions and numerical or experimental results for the variation of cumulated MI gain as a function of the frequency spacing among the pumps.

\section{CONCLUSION}

In this work we have demonstrated the generation of PMI sidebands in the normal dispersion regime of a relatively long span of dispersion-compensating fiber with relatively low PMD. PMI is induced by XPolM among orthogonally polarized and intense quasi-cw WDM pumps. We have presented an extensive investigation of the pump power and pump frequency spacing dependence of the PMI gain, with excellent quantitative agreement with numerical solutions of the Manakov system. We have also shown that for a pump spacing larger than about $150 \mathrm{GHz}$, cascade FWM leads to a nonlinear saturation of the PMI gain. This work opens the way to further studies devoted to exploiting the relatively large PMI gain for parametric signal amplification. We envisage that the present results may have significant consequences for the design of nonlinear transmission systems, and may have important applications to optical signal processing devices and fiber lasers. Moreover our observations may impact the application of the Manakov model to describe the formation of extreme waves in multicomponent systems [29,30].

\section{ACKNOWLEDGMENTS}

The authors thank P. Tchofo Dinda for stimulating discussions. We gratefully acknowledge support from the French National Research Agency through Labex ACTION program (Contract No. ANR-11-LABX-0001-01), "NoAWE" project (Grant No. ANR-14-ACHN-0014-01) and "OptiRoC" project (Grant No. ANR-12-BS04-0011). We also acknowledge the financial support from the Italian Ministry of University and Research (MIUR, Project No. 2012BFNWZ2), the Conseil Régional de Bourgogne through the Photcom PARI grant, the iXCore Foundation, and the European Research Council under Grant Agreement No. 306633, ERC PETAL.

[1] C. Sulem and P.-L. Sulem, The Nonlin-
ear Schrödinger Equation: Self-focusing and $\quad$ Collapse $\quad$ (Springer-Verlag, New York,


[2] F. Baronio, A. Degasperis, M. Conforti, and S. Wabnitz, Solutions of the Vector Nonlinear Schrödinger Equations: Evidence for Deterministic Rogue Waves, Phys. Rev. Lett. 109, 044102 (2012).

[3] M. Onorato, A. R. Osborne, and M. Serio, Modulational Instability in Crossing Sea States: A Possible Mechanism for the Formation of Freak Waves, Phys. Rev. Lett. 96, 014503 (2006).

[4] Y. V. Bludov, V. V. Konotop, and N. Akhmediev, Vector rogue waves in binary mixtures of Bose-Einstein condensates, Eur. Phys. J: Spec. Top. 185, 169 (2010).

[5] V. G. Ivancevic, Adaptive-wave alternative for the BlackScholes option pricing model, Cognit. Comput. 2, 17 (2010).

[6] S. V. Manakov, On the theory of two-dimensional stationary self-focusing of electromagnetic waves, Zh. Eksp. Teor. Fiz. 65, 505 (1973) [Sov. Phys. J. Exp. Theor. Phys. 38, 248 (1974)].

[7] Y. S. Kivshar and G. P. Agrawal, Optical Solitons: From Fibers to Photonic Crystals (Academic Press, San Diego, 2003).

[8] P. G. Kevrekidis, D. J. Frantzeskakis, and R. CarreteroGonzalez, Emergent Nonlinear Phenomena in Bose-Einstein Condensates (Springer-Verlag, Berlin, 2008).

[9] M. Winter, C.-A. Bunge, D. Setti, and K Petermann, A statistical treatment of cross-polarization modulation in DWDM systems, J. Lightwave Technol. 27, 3739 (2009).

[10] S. G. Evangelides, L. F. Mollenauer, J. P. Gordon, and N. S. Bergano, Polarization multiplexing with solitons, J. Lightwave Technol. 10, 28 (1992).

[11] F. Yaman and G. Li, Nonlinear impairment compensation for polarization-division multiplexed WDM transmission using digital backward propagation, IEEE Photonics J. 1, 144 (2009).

[12] S. Mumtaz, R.-J. Essiambre, and G. P. Agrawal, Nonlinear propagation in multimode and multicore fibers: generalization of the Manakov equations, J. Lightwave Technol. 31, 398 (2013).

[13] This is not a necessary condition, as another condition exists which leads to integrability of the coupled NLSEs: V. E. Zakharov and E. I. Schulman, To the integrability of the system of two coupled nonlinear Schrödinger equations, Physica D 4, 270 (1982).

[14] G. P. Agrawal, Nonlinear Fiber Optics, 5th ed. (Academic Press, San Diego, 2013).

[15] P. K. A. Wai and C. R. Menyuk, Polarization mode dispersion, decorrelation, and diffusion in optical fibers with randomly varying birefringence, J. Lightwave Technol. 14, 148 (1996).

[16] D. Marcuse, C. R. Menyuk, and P. K. A. Wai, Application of the Manakov-PMD equation to studies of signal propagation in optical fibers with randomly varying birefringence, J. Lightwave Technol. 15, 1735 (1997).

[17] J. U. Kang, G. I. Stegeman, J. S. Aitchison, and N. Akhmediev, Observation of Manakov Spatial Solitons in AlGaAs Planar Waveguides, Phys. Rev. Lett. 76, 3699 (1996).

[18] P. L. Christiansen, J. C. Eilbeck, V. Z. Enolskii and N. A. Kostov, Quasi-periodic and periodic solutions for coupled nonlinear Schrodinger equations of Manakov type, Proc. R. Soc. London, Ser. A 456, 2263 (2000).

[19] M. G. Forest, D. W. McLaughlin, D. J. Muraki, and O. C. Wright, Nonfocusing instabilities in coupled, integrable nonlinear Schrödinger pdes, J. Nonlinear Sci. 10, 291 (2000).

[20] O. C. Wright III, On elliptic solutions of a coupled nonlinear Schrödinger system, Physica D 264, 1 (2013).
[21] A. M. Kamchatnov, Periodic waves in a two-component BoseEinstein condensate, JETP 118, 630 (2014).

[22] Z. Chen, M. Segev, T. H. Coskun, D. N. Christodoulides, and Y. S. Kivshar, Coupled photorefractive spatial-soliton pairs, J. Opt. Soc. Am. B 14, 3066 (1997).

[23] C. Hamner, J. J. Chang, P. Engels, and M. A. Hoefer, Generation of Dark-Bright Soliton Trains in Superfluid-Superfluid Counterflow, Phys. Rev. Lett. 106, 065302 (2011).

[24] M. A. Hoefer, J. J. Chang, C. Hamner, and P. Engels, Dark-dark solitons and modulational instability in miscible two-component Bose-Einstein condensates, Phys. Rev. A 84, 041605(R) (2011).

[25] L. Beygi, E. Agrell, J. M. Kahn, and M. Karlsson, Coded modulation for fiber-optic networks: Toward better tradeoff between signal processing complexity and optical transparent reach, IEEE Signal Process. Mag. 31, 93 (2014).

[26] J. Fatome, I. El-Mansouri, J. L. Blanchet, S. Pitois, G. Millot, S. Trillo, and S. Wabnitz, Even harmonic pulse train generation by cross-polarization-modulation seeded instability in optical fibers, J. Opt. Soc. Am. B 30, 99 (2013).

[27] J. Roske, Some nonlinear multiphase reactions, Stud. Appl. Math. 55, 231 (1976).

[28] C. K. Law, C. M. Chan, P. T. Leung, and M.-C. Chu, Critical velocity in a binary mixture of moving Bose condensates, Phys. Rev. A 63, 063612 (2001).

[29] F. Baronio, M. Conforti, A. Degasperis, S. Lombardo, M. Onorato, and S. Wabnitz, Vector Rogue Waves and Baseband Modulation Instability in the Defocusing Regime, Phys. Rev. Lett. 113, 034101 (2014).

[30] F. Baronio, S. Chen, P. Grelu, S. Wabnitz, and M. Conforti, Baseband modulation instability as the origin of rogue waves, Phys. Rev. A 91, 033804 (2015).

[31] V. I. Bespalov and V. I. Talanov, Filamentary structures of light beams in nonlinear liquids, J. Exp. Theor. Phys. Lett. 3, 307 (1966).

[32] T. B. Benjamin and J. E. Feir, The designation of wavetrains on deep water, Part 1, Theory, J. Fluid. Mech. 27, 417 (1967).

[33] K. Tai, A. Hasegawa, and A. Tomita, Observation of Modulational Instability in Optical Fibers, Phys. Rev. Lett. 56, 135 (1986).

[34] A. L. Berkhoer and V. E. Zakharov, Self excitation of waves with different polarizations in nonlinear media, Zh. Eksp. Teor. Fiz. 58, 903 (1970) [Sov. Phys. J. Exp. Theor. Phys. 31, 486 (1970)].

[35] J. E. Rothenberg, Modulational instability for normal dispersion, Phys. Rev. A 42, 682 (1990).

[36] P. D. Drummond, T. A. B. Kennedy, J. M. Dudley, R. Leonhardt, and J. D. Harvey, Cross-phase modulational instability in high-birefringence fibers, Opt. Commun. 78, 137 (1990)

[37] E. Seve, P. Tchofo Dinda, G. Millot, M. Remoissenet, J. M. Bilbault, and M. Haelterman, Modulational instability and critical regime in a highly birefringent fiber, Phys. Rev. A 54, 3519 (1996).

[38] G. P. Agrawal, Modulation Instability Induced by Cross-Phase Modulation, Phys. Rev. Lett. 59, 880 (1987).

[39] J. E. Rothenberg, Modulational Instability of Copropagating Frequencies for Normal Dispersion, Phys. Rev. Lett. 64, 813 (1990). 
[40] G. P. Agrawal, Agrawal Replies on the Comment of J. E. Rothenberg, Phys. Rev. Lett. 64, 814 (1990).

[41] M. Yu, C. J. McKinstrie, and G. P. Agrawal, Instability due to cross-phase modulation in the normal-dispersion regime, Phys. Rev. E 48, 2178 (1993).

[42] Y. Frignac, G. Charlet, W. Idler, R. Dischler, P. Tran, S. Lanne, S. Borne, C. Martinelli, G. Veith, A. Jourdan, J. P. Hamaide, and S. Bigo, Transmission of 256 wavelength-division and polarization division-multiplexed channels at $42.7 \mathrm{~Gb} / \mathrm{s}(10.2 \mathrm{~Tb} / \mathrm{s}$ capacity) over $3 \times 100 \mathrm{~km}$ of TeraLight ${ }^{\mathrm{TM}}$ fiber, in Proceedings of Optical Fiber Communication Conference and Exhibit, 2002 (IEEE, New York, 2002), pp. FC5-1-FC5-3.

[43] A. H. Gnauck, G. Charlet, P. Tran, P. J. Winzer, C. R. Doerr, J. C. Centanni, E. C. Burrows, T. Kawanishi, T. Sakamoto, and K. Higuma, 25.6-Tb/s WDM transmission of polarizationmultiplexed RZ-DQPSK signals, J. Lightwave Technol. 26, 79 (2008).

[44] X. Liu, F. Buchali, and R. W. Tkach, Improving the nonlinear tolerance of polarization-division-multiplexed CO-OFDM in long-haul fiber transmission, J. Lightwave Technol. 27, 3632 (2009).
[45] E. F. Mateo, X. Zhou, and G. Li, Selective post-compensation of nonlinear impairments in polarization-division multiplexed WDM systems with different channel granularities, IEEE J. Quantum Electron. 47, 109 (2011).

[46] A. Hasegawa, Generation of a train of soliton pulses by induced modulational instability in optical fibers, Opt. Lett. 9, 288 (1984).

[47] P. V. Mamyshev, S. V. Chernikov, and E. M. Dianov, Generation of fundamental soliton trains for high-bit-rate optical fiber communication lines, IEEE J. Quantum Electron. 27, 2347 (1991).

[48] J. M. Dudley, G. Genty, and S. Coen, Supercontinuum generation in photonic crystal fiber, Rev. Mod. Phys. 78, 1135 (2006).

[49] D. R. Solli, C. Ropers, P. Koonath, and B. Jalali, Optical rogue waves, Nature 450, 1054 (2007).

[50] B. Kibler, J. Fatome, C. Finot, G. Millot, F. Dias, G. Genty, N. Akhmediev, and J. M. Dudley, The Peregrine soliton in nonlinear fibre optics, Nat. Phys. 6, 790 (2010).

[51] A. Chabchoub, N. P. Hoffmann, and N. Akhmediev, Rogue Wave Observation in a Water Wave Tank, Phys. Rev. Lett. 106, 204502 (2011). 